http://www.jfas.info

\title{
DESIGN AN AUTOMATIC TEMPERATURE CONTROL SYSTEM BASED ON PIC CONTROLLER FOR SMART VENTILATION FAN
}

\author{
N. N. S. N. Dzulkefli ${ }^{1}$, R. Abdullah ${ }^{1}$, A. N. Jaafar ${ }^{1}$, R. Shafie ${ }^{1}$, I. M. Yassin ${ }^{2,}$, Z. I. \\ Rizman $^{1}$ and H. Z. Abidin ${ }^{2}$
}

\begin{abstract}
${ }^{1}$ Faculty of Electrical Engineering, UniversitiTeknologi MARA, 23000 Dungun, Terengganu, Malaysia

${ }^{2}$ Faculty of Electrical Engineering, UniversitiTeknologi MARA, 40450 Shah Alam, Selangor, Malaysia
\end{abstract}

Published online: 10 September 2017

\begin{abstract}
This paper presents an inventive prototype design of ventilation fan with smart features. It uses a microcontroller to create an automation function. The ventilation fan operates automatically according to the temperature which set by the user. This is to ensure the cooling process, especially for large space and also in hot weather due to global warming works well and effectively. An LM35 temperature sensor is placed in the room to sense the room temperature. The PIC16F887 microcontroller circuit will control the ventilation fan based on temperature detected by the sensor. Users initially set the temperature to $\mathrm{ON}$ the fan together with overheat temperature to $\mathrm{ON}$ the buzzer. If the room's temperature is more than set value, the ventilation fan will function while the buzzer will produce sound when it is high than the set value. Thus, through this circuit, it is easy to use and make human's life simpler and better.
\end{abstract} Keywords: microcontroller; temperature sensor; PIC16F887.

Author Correspondence, e-mail: ihsan.yassin@gmail.com doi: http://dx.doi.org/10.4314/jfas.v9i3s.60 


\section{INTRODUCTION}

In this day and age, automatic appliances are one of the important signs in our country [14]. An automatic washing machine is the first automatic facility come into people's home [13]. Then followed by air-condition, cooler, various control systems based on android smartphone and so forth [1]. There does always exist the basic of the new technology features every year especially in today's globalization era. The automatic temperature control system becomes the most popular features which quickly achieved its popularity due to its importance to certain applications [2]. Applications which require temperature control are frequently become more important in industry today [3].

Malaysia is known for its hot weather. This issue may give a problem for human, especially to control the hot air inside the building because it tends to make the temperature of the surroundings become high. To overcome this problem, human nowadays mostly uses a ventilation fan that is more economical compared to air conditioners that use a lot of electricity. So, an automatic temperature control system is needed for the controlling purpose in the ventilation fan according to the temperature changes [4]. Ventilating is the process of changing or replacing air in any space to control temperature or eliminate moisture, smoke, dust, unkind smells, microorganisms and so on. Currently, the demand for accurate temperature control and air ventilation control has conquered many of industrial domains. For examples, the heat process, alimentary industry, automotive, industrial spaces or office buildings where the air should be always cool in order to maintain a comfortable environment for its residents [3].

In the last few years, research has been done regarding to temperature control for different types of processes. For example, a low cost application for temperature control in a ventilation system using the PIC18F4620 was designed and developed [3], an automatic control solution is suggested to control the fan speed with LM35DZ temperature sensor, PIC16F877A microcontroller, brush less DC motor and few of electronic components is designed and implemented to control the fan speed automatically [4], a microcontroller-based fuzzy logic controller is introduced to stabilize at the desired temperature, while sudden changes in temperature can be severe [5], automatic smart kitchen system using LM35 and 
smoke detector that controlled by PIC16F877A which is advancements system form air ventilation to discharge heat and smoke at kitchen [2], the design and simulation of temperature control of chamber based on automatic fan speed control with LM35 temperature sensor and 8951 microcontroller [6], the development of a fuzzy inference system that effectively and intelligently controls the rotating speed of an electric fan according to the temperature of environment and its relative humidity [7], the design of a room temperature and humidity controller using fuzzy logic [8], design an automatic temperature control system for Smart Electric Fan Using PIC16F876A [9], the motor speed control based on temperature using Controller Area Network (CAN) protocol [10], design and implement an automated temperature control system using embedded system design in order to control the temperature of a system by using LM35 temperature sensor, PIC AT89c52 $\mu$ C, Liquid Crystal Display (LCD) module, switching device, driver, Pulse Width Modulation (PWM) generator, a fan and a heater [11] and the development and temperature control of smart egg incubator system for various types of egg [12]. Thus, this paper will show how PIC16F887 microcontrollers can be used and applied in a real-world situation. It will use a microcontroller in a temperature control system that can be used to control a room temperature by using ventilation fan to regulate the temperature back to normal.

This paper proposes a smart ventilation fan by using an LM35 temperature sensor. The user will switch $\mathrm{ON}$ the ventilation fan when the temperature inside the building or in the house exceeds the set temperature. The temperature can be set in order for the LM35 sensor to detect different value of temperature changes in surroundings, depends on temperature needed for the situation. So, with this smart ventilation fan, temperature of air can be controlled and regulated automatically. The ventilation fan will function automatically after the temperature sensor detects the temperature in surroundings that exceeds the temperature that set before.

Additionally, this smart ventilation fan also can set the overheat temperature that will warn the user if the temperature is over than overheat temperature settings earlier. It is to ensure the safety of the houses or buildings from anything bad happen. The speed of the fan also can be set to rotate about $50 \%$ or $100 \%$. They are all depends on the user when key in the desired temperature, overheat temperature and also the fan's speed. 


\section{METHODOLOGY}

This project presents the design and development of the automatic temperature control system applicable for smart ventilation fan. Ventilation fan is said to be smart because it will work according to a certain temperature. This idea arises from a problem faced by hot weather. Therefore, the smart ventilation fan is the best solution to overcome this problem, especially the places that cannot use the air conditioner as bathrooms and kitchen. Besides, the ventilation fan is cheaper and low power consumption than air conditioner. The block diagram of the system is shown in Fig. 1. The integrated system of smart ventilation fan consists of a temperature sensor LM35 for input, the PIC16F887 microcontroller for data intepretation and ventilation fan and buzzer as the outputs. The LM35 sensor is for input detection. The microcontroller uses the information provided by the sensor to control the ventilation fan and buzzer.

Input Controller

Output

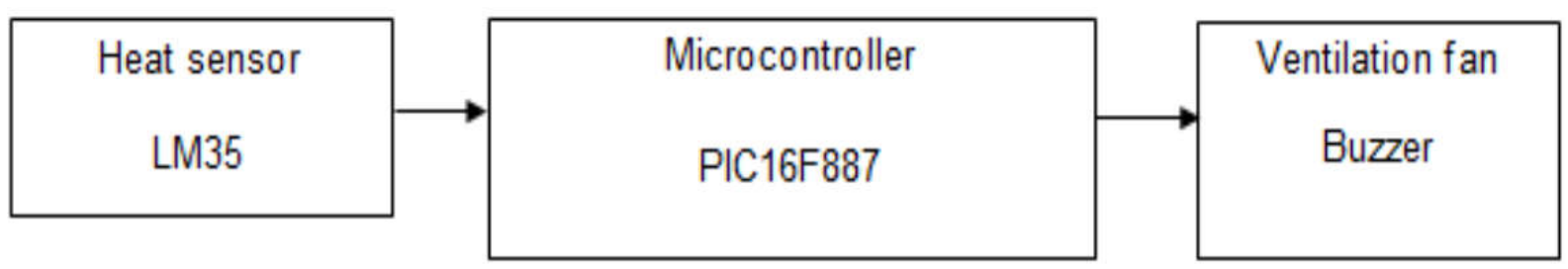

Fig.1. Block diagram of the smart ventilation fan system

This project consists of two major parts, which are hardware and software design and development. In the hardware design, this project is built like a room in a house as its prototype. 


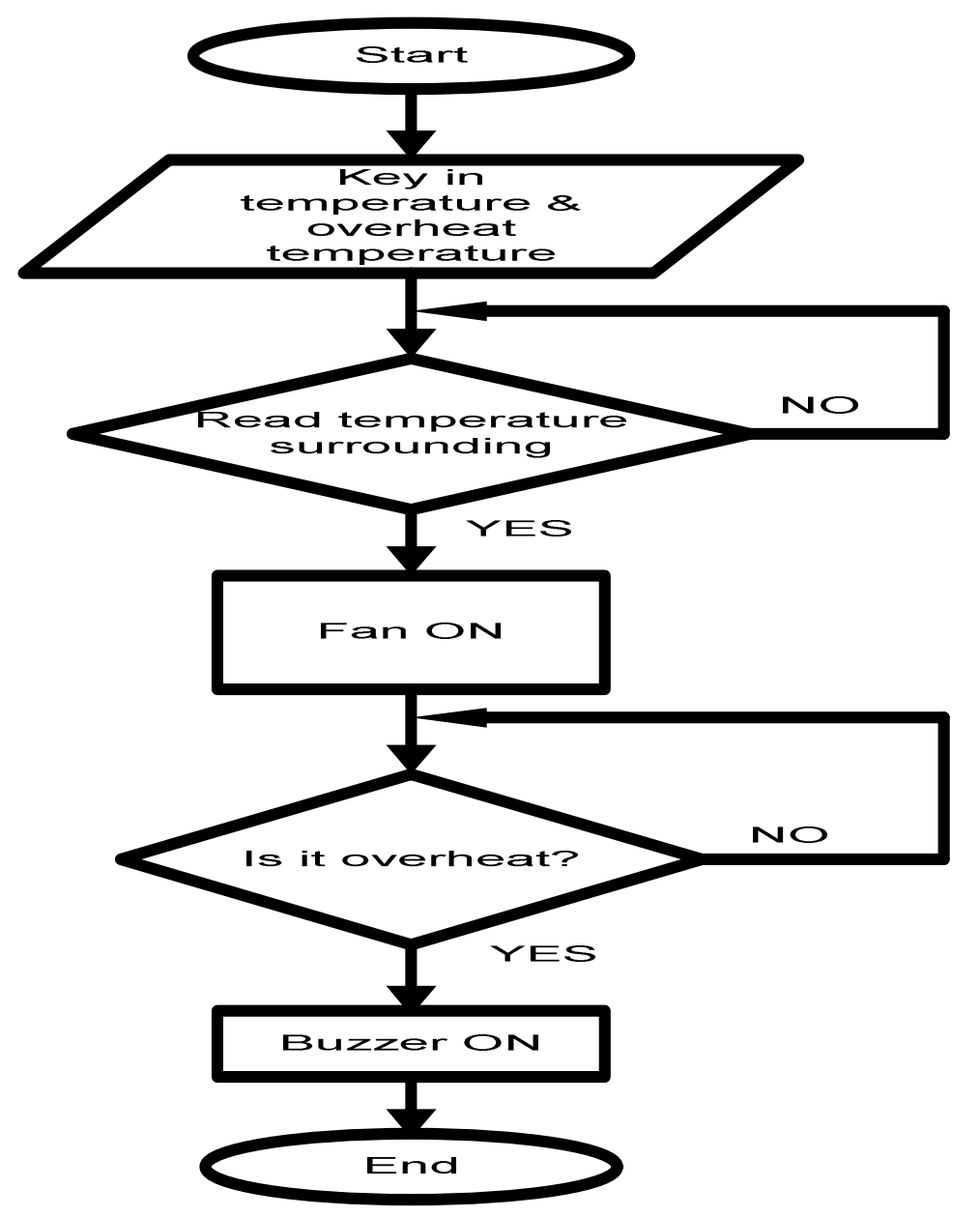

Fig.2. Flowchart of the smart ventilation fan system

Fig. 2 shows the flowchart the smart ventilation fan system. Users firstly will key in the desired temperature and overheat temperature to $\mathrm{ON}$ the fan and the buzzer automatically. The sensor will detect the surrounding temperature and hence LCD will show the reading of the temperature. This project is using microcontroller as a brain to control the fan according to temperature variation. The system measures the temperature which detected by the sensor LM35, where it will control the fan according to the temperature settings in programming. In the software development, the procedure is developed and simulated by using the Proteus 6.8 Lite software on a virtual circuit before it is uploaded and implemented using PIC16F887. The system indicates the temperature from the PIC16F887. Then, the temperature is compared with the setting value. When the temperature in the room is more than the desired value, the fan will automatically $\mathrm{ON}$ and the LCD will display the temperature. If the temperature in the room is more than the overheat temperature which is setting earlier, the buzzer will automatically ON. 


\section{RESULTS AND DISCUSSION}

The results for this project are divided into two parts, which are software simulation and actual hardware. In software simulation, the automatic temperature control system for ventilation fan is function according to the procedure. Fig. 3 shows the complete design of the smart ventilation fan circuit in Proteus simulation. This circuit is supplied by $12 \mathrm{~V}$ direct current (DC) supply. After the $12 \mathrm{~V}$ DC supply is turned ON, the circuit starts to operate. It is connected to voltage regulator circuit to produce $5 \mathrm{~V}$ voltage. Then, the sensor starts its function to measure the changes of temperature surrounds the area in the room. The PIC16F887 is the main module of the circuit. All the operations are controlled by this module to produce the output.

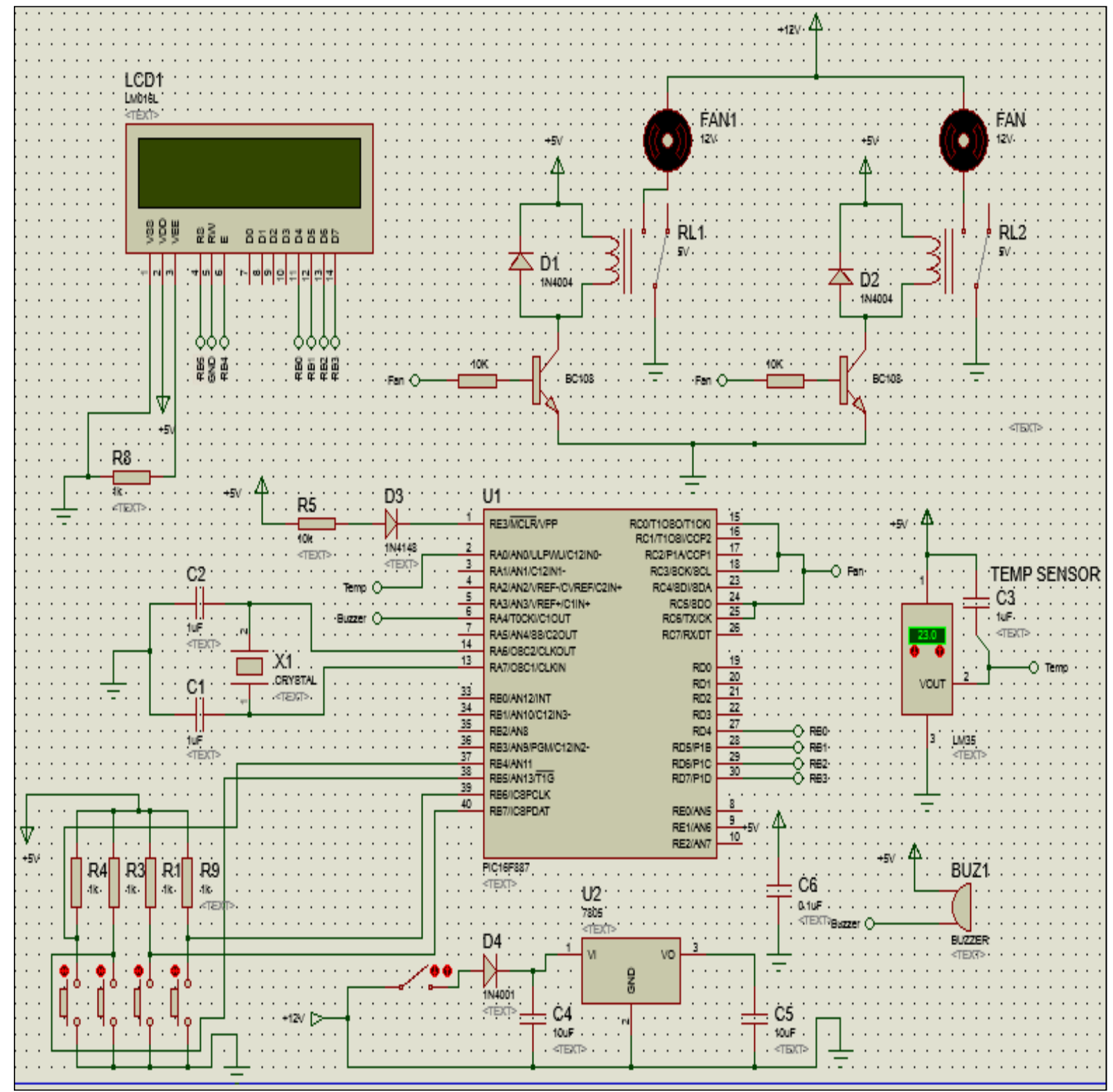


Fig.3. Schematic diagram of smart ventilation fan

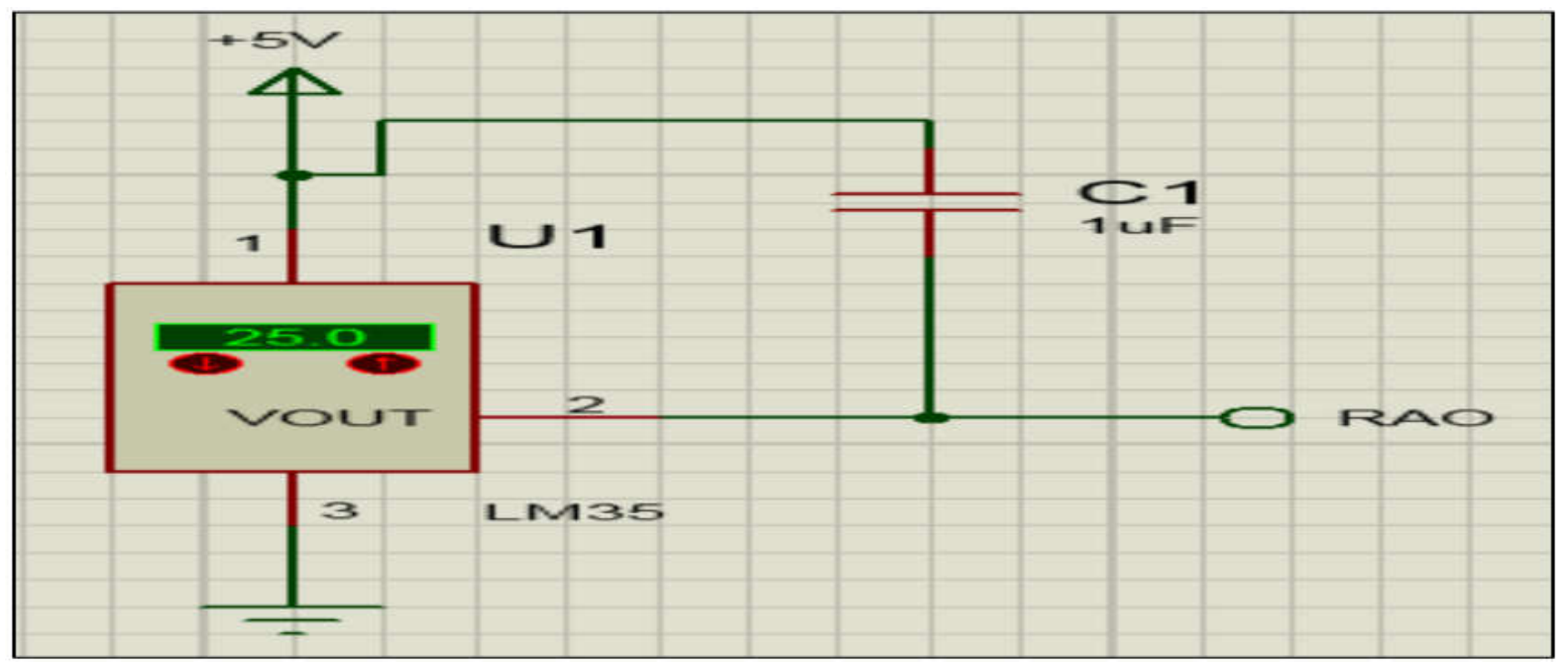

Fig.3(a). The temperature in the room is lower than desired temperature

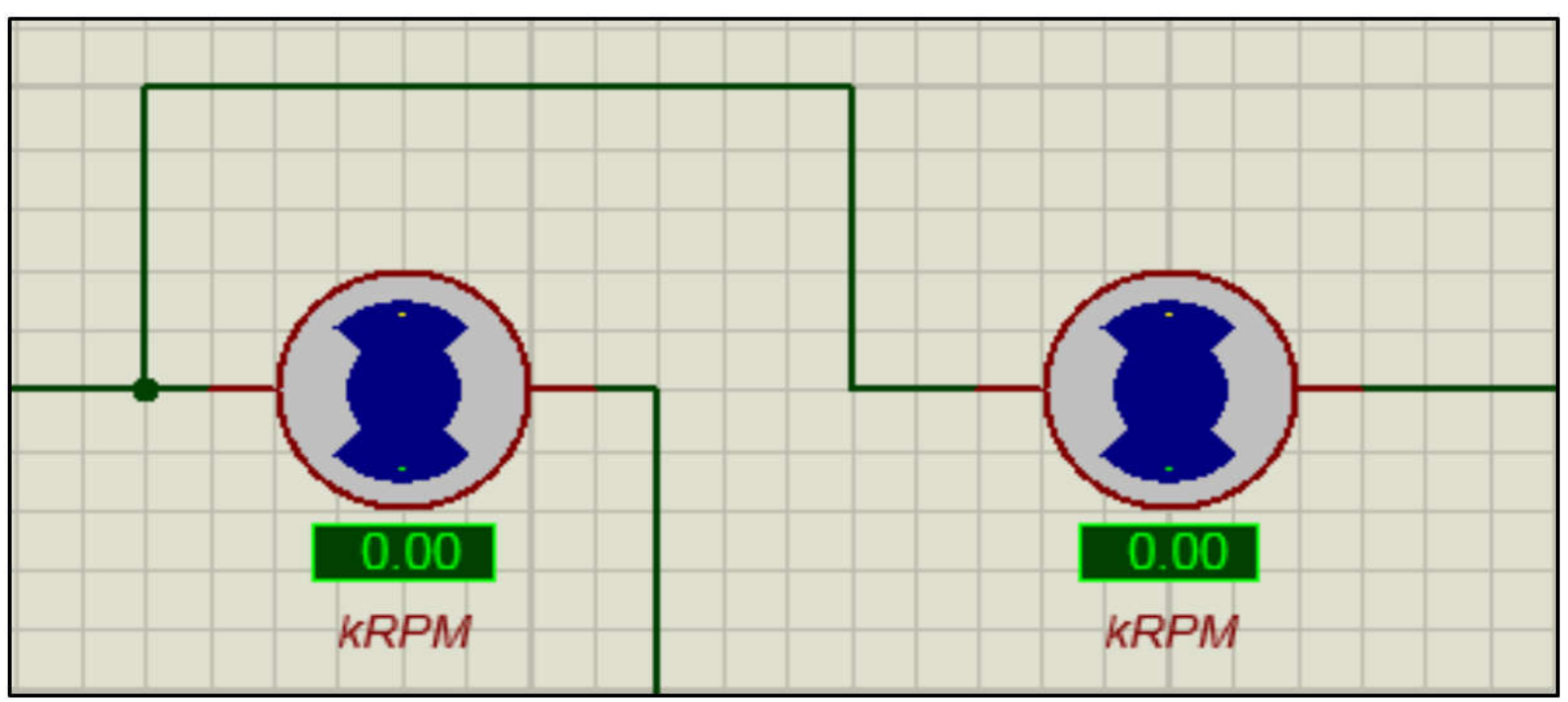

Fig.3(b). The smart ventilation fan will OFF when the temperature in the room is lower than desired temperature

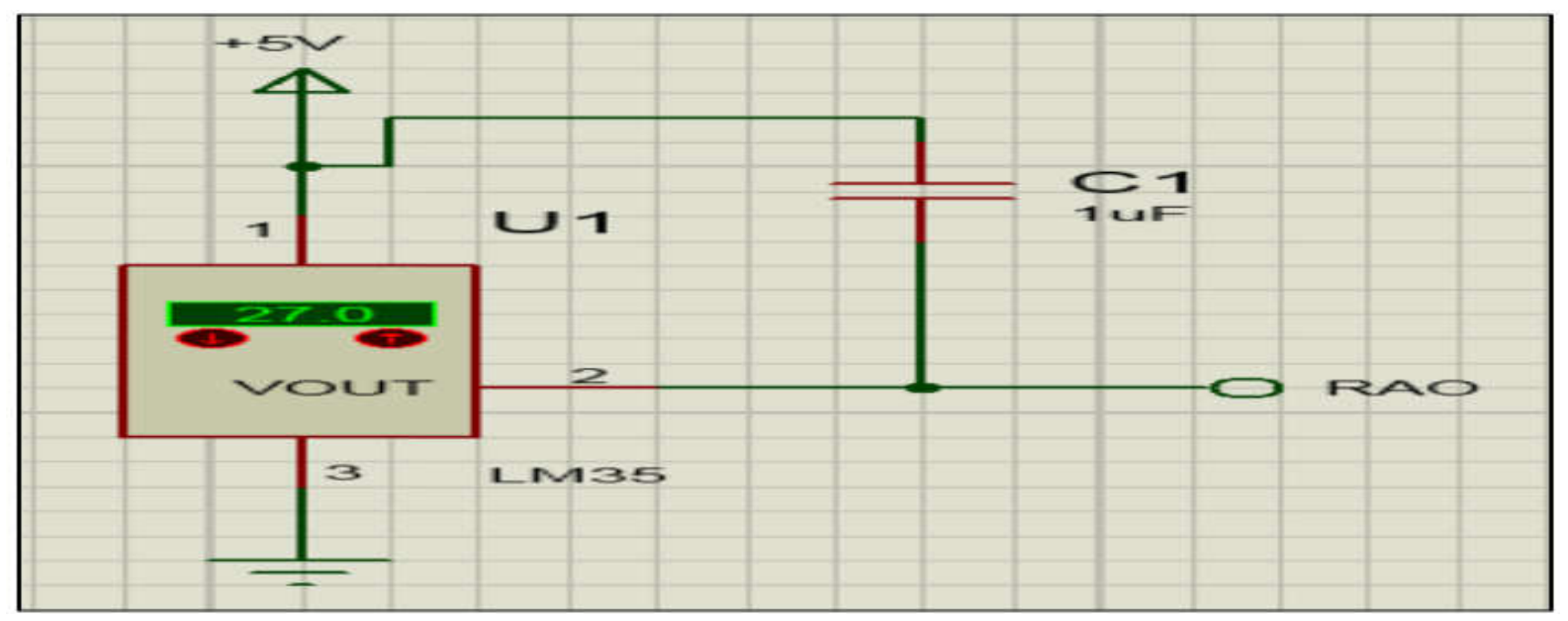


Fig.4(a). The temperature in the room is higher than desired temperature

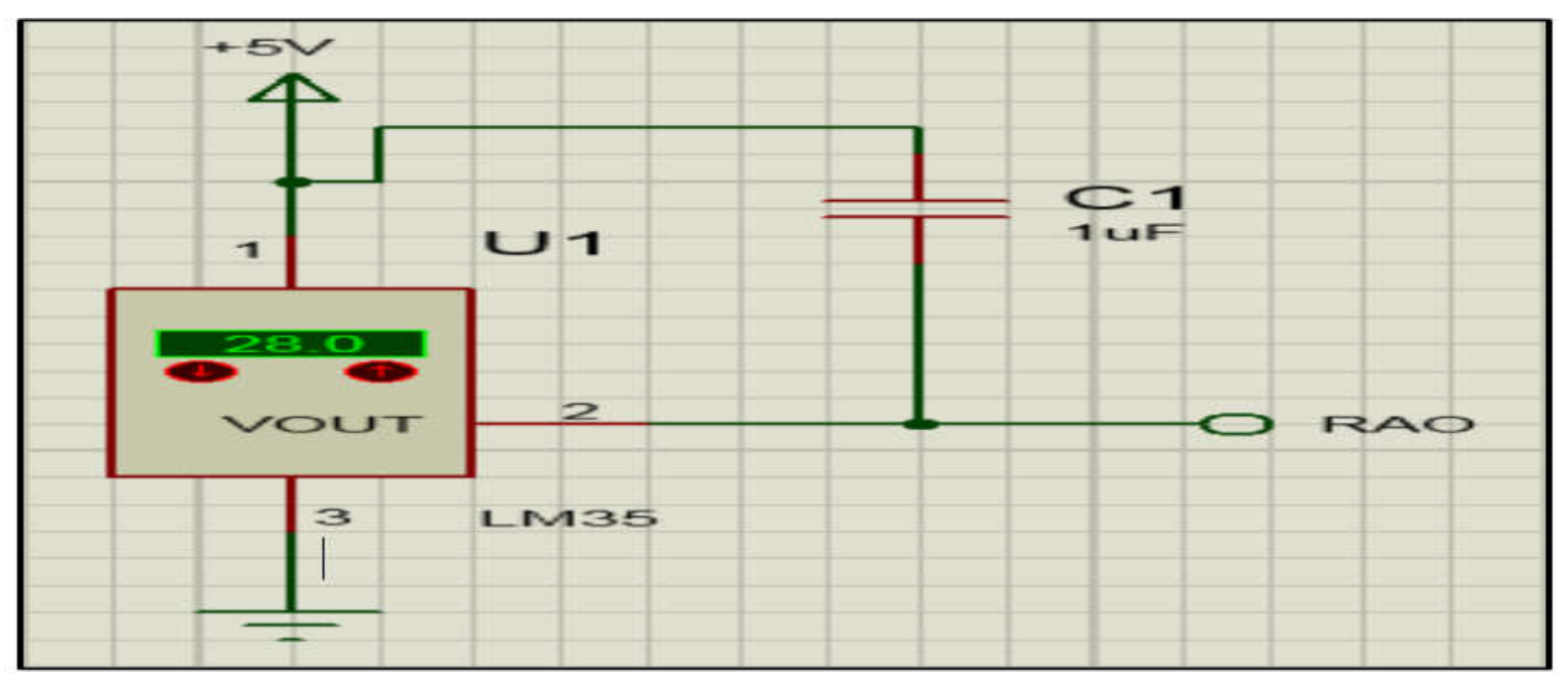

Fig.4(b). The temperature in the room is higher than desired temperature

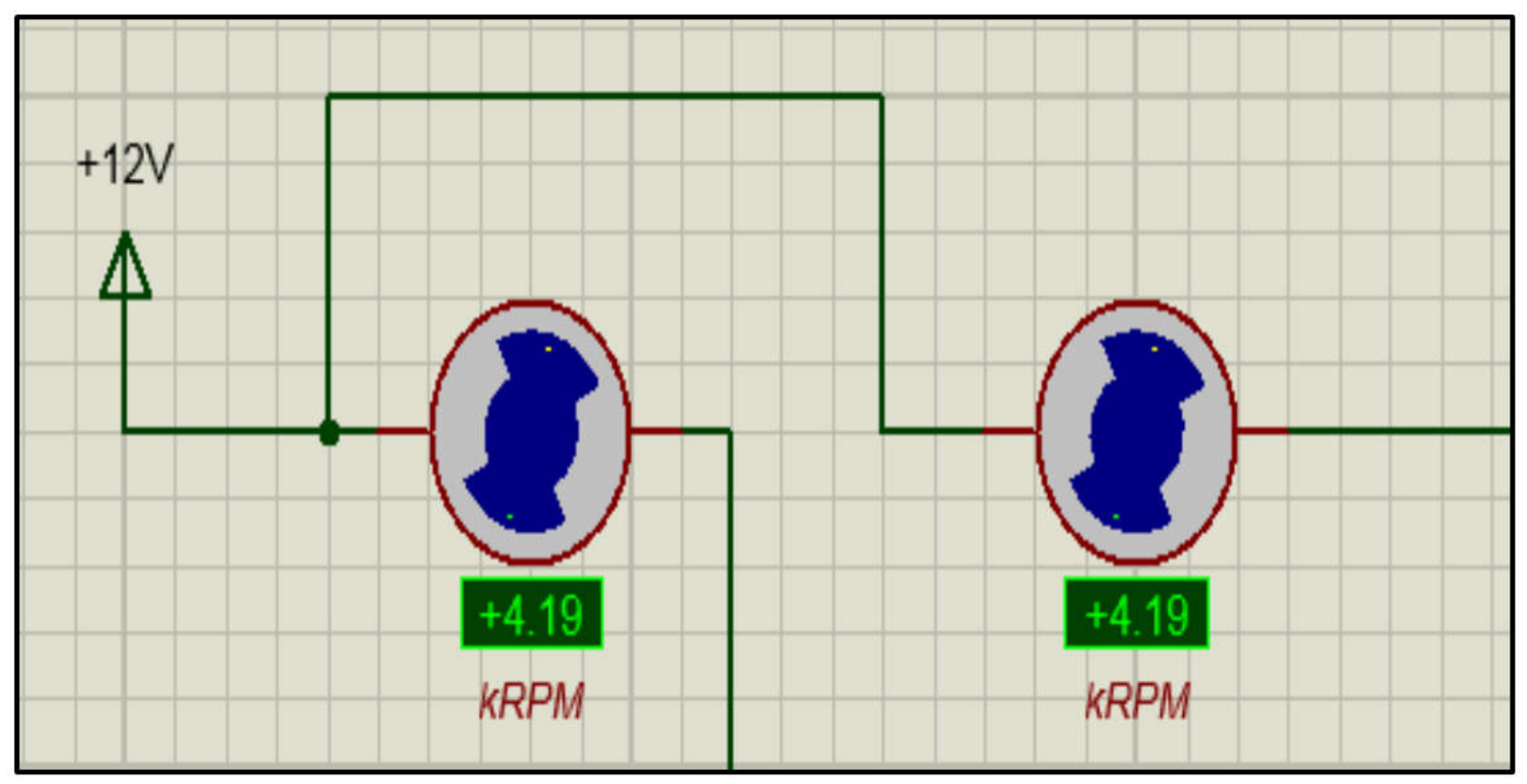

Fig.4(c). The smart ventilation fan will $\mathrm{ON}$ when the temperature in the room is lower than desired temperature

The hardware part is shown in Fig. 5 and Fig. 6. The temperature sensor will detect the temperature from the surrounding in the room while PIC16F887, which is the main module in this circuit will measure the temperature. This circuit using LM35 as temperature sensor whose range is wider than common temperature sensor. LM35 is an accurate IC temperature sensor, whose output voltage is proportional to the temperature [2]. The LM35 temperature sensor has a linear $+10.0 \mathrm{mV} /{ }^{\circ} \mathrm{C}$ scale factor and a temperature range from $-55^{\circ} \mathrm{C}$ to $+150^{\circ} \mathrm{C}$ [6]. The microcontroller PIC16F887 is programmed to response to the sensor when it detects 
the temperature which is higher than desired temperature. The smart ventilation fan will $\mathrm{ON}$ together with the buzzer if the detected temperature is more than overheat temperature, which also setting earlier. Fig. 3(a) and 3(b) show the result when the temperature in the room is lower than the setting temperature, the smart ventilation fan will OFF. If the temperature is set at $27^{\circ} \mathrm{C}$, the ventilation fan will automatically $\mathrm{ON}$ when the temperature surroundings detects by LM35 reached $27^{\circ} \mathrm{C}$ or above as shown in Fig. 4(a), 4(b) and 4(c). Then, if overheat temperature is set at $40^{\circ} \mathrm{C}$, the buzzer will automatically $\mathrm{ON}$ when the LM35 detects the surrounding temperature at about $40^{\circ} \mathrm{C}$ or above.

Overall, this smart ventilation fan will automatically regulate the temperature surroundings in some places such as buildings and houses. Table 1 shows the results for the operation of the automatic temperature control for the smart ventilation fan after the implementation of the programming.

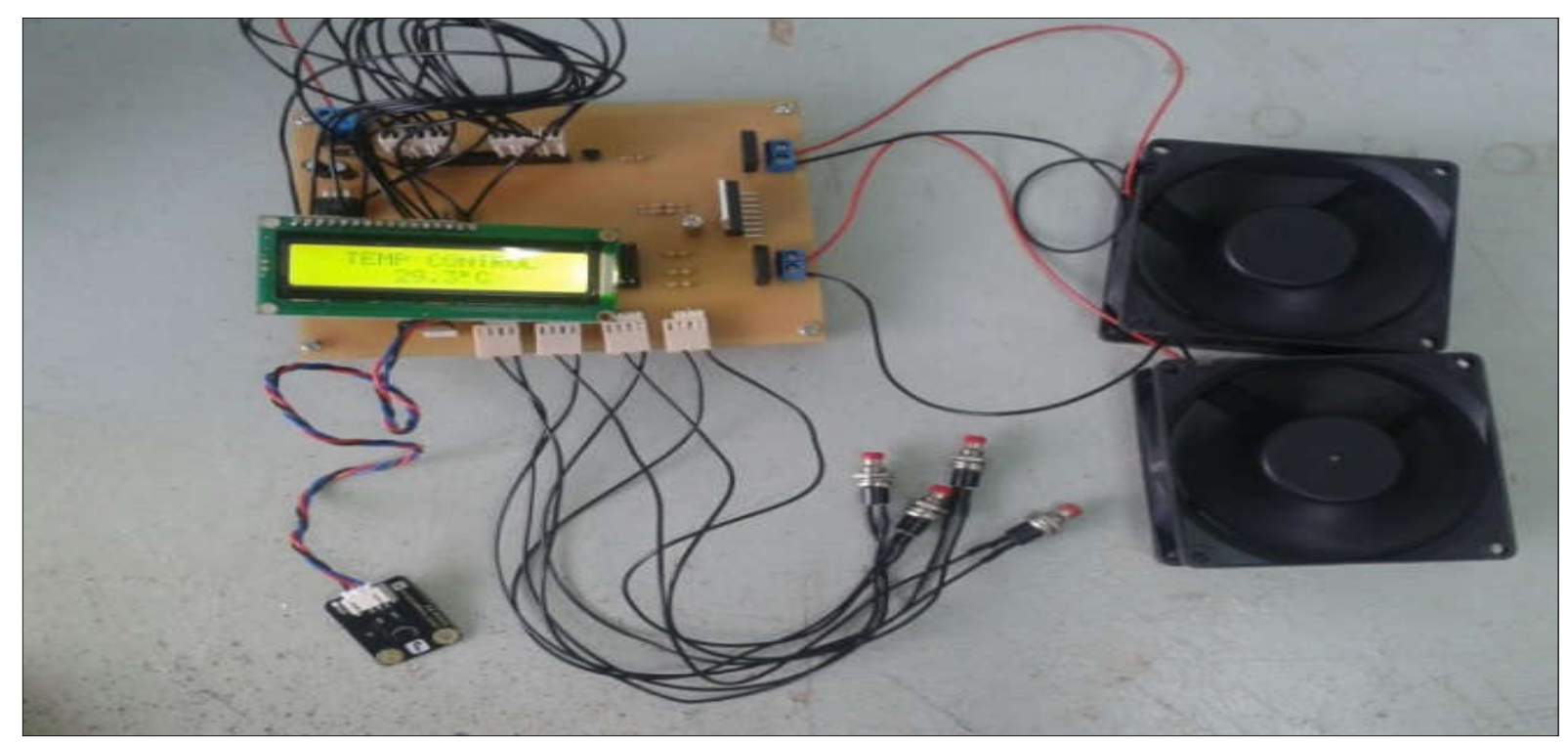

Fig.5. Circuit simulation of smart ventilation fan on PCB board 


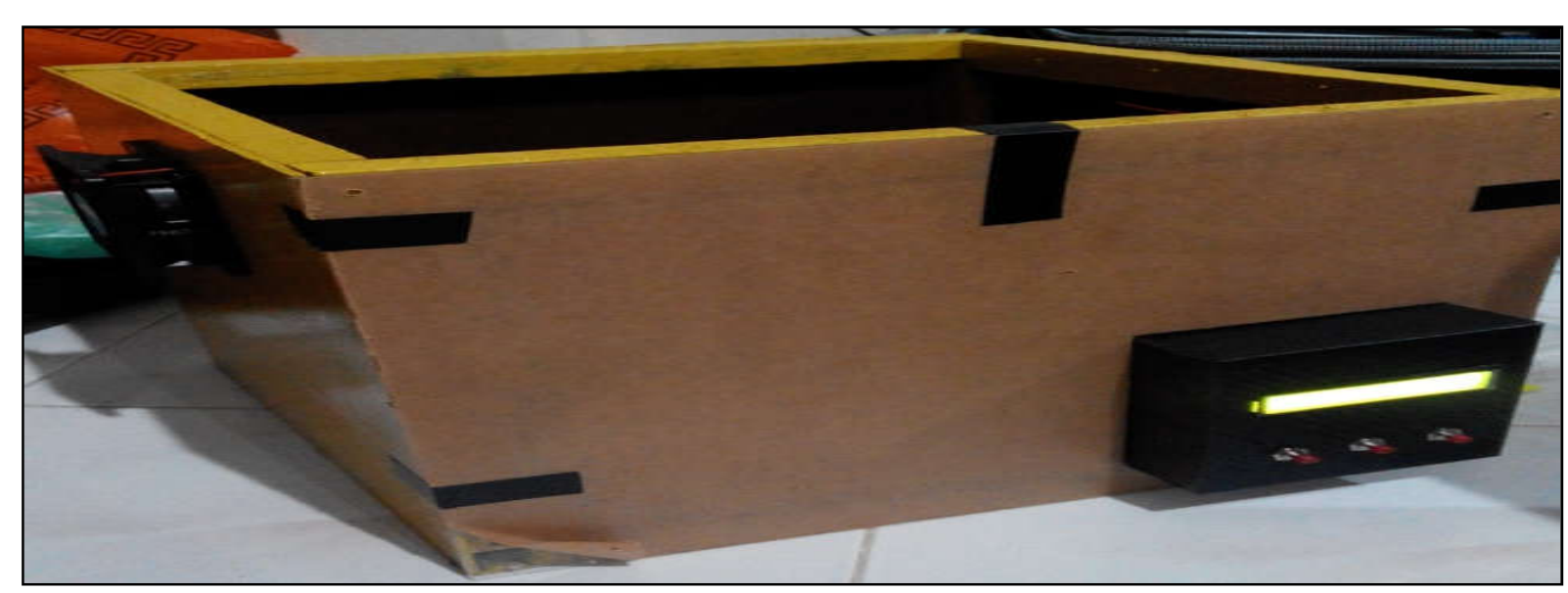

Fig.6. Prototype of smart ventilation fan

Table 1. Results operations of smart ventilation fan

\begin{tabular}{ccccc}
\hline \multicolumn{2}{c}{ Set Temperature } & Surrounding Temperature & Fan & Buzzer \\
Temperature & Overheat & $\left({ }^{\circ} \mathbf{C}\right)$ Detect by LM35 & & \\
$\left({ }^{\circ} \mathbf{C}\right)$ & Temperature $\left({ }^{\circ} \mathbf{C}\right)$ & & & \\
\hline 27 & 40 & 25 & OFF & OFF \\
27 & 40 & 27 & ON & OFF \\
27 & 40 & 28 & ON & OFF \\
\hline
\end{tabular}

\section{CONCLUSION}

In conclusion, the developing of automatic temperature control using LM35 which is controlled by PIC16F887 for smart ventilation fan is done very well. The hardware implementation together with its operation work accordingly as stated in the procedure. This project has fulfilled the main objectives, which is to ease the human in their daily life. Besides that, at the same time this smart ventilation fan will control temperature surroundings automatically as well as to ensure that the cooling process in the room or houses works well and effectively. Ventilation fan is function to force the hot air inside to be drawn out from the building or houses. So, the cooling process for the whole room or houses works efficiently. By using this smart ventilation fan, the temperature in the house or room can be regulated automatically.

In the future, there is an improvement can be made in order to upgrade the features of this project such as adding an infrared sensor to assist the user to adjust the temperature. The 
sensor is to be built as a remote control to the smart ventilation fan. So, when the user wants to adjust the temperature, they only need to use the remote control from a long distance about three to four meters from the ventilation fan to adjust the setting temperature.

\section{ACKNOWLEDGEMENTS}

The authors would like to graciously acknowledge the Ministry of Higher Education and UniversitiTeknologi MARA for supporting this research work through Grant No: FRGS/1/2015/ICT03/UiTM/02/4.

\section{REFERENCES}

[1] Chapke S S, Markande S D. Design of energy efficient smart fan. In 44th IRF International Conference, 2015

[2] Hadi N A A, Hasan M H C, Hashim N M Z, Salleh A, Salam A A, Hasan Z. Automatic smart kitchen system using microcontroller. International Journal for Advance Research in Engineering and Technology, 2015, 3(6):30-34

[3] Levarda B, Budaciu C. The design of temperature control system using PIC18F4620. 2010, http://www.ace.tuiasi.ro/users/103/14_F4_10_Levarda.pdf

[4] Mustafa S, Hossam A, Muammer M. Automatic fan speed control system using microcontroller. In 6th International Conference on Electrical, Electronics and Civil Engineering, 2014, pp. 86-89

[5] Jebelli A, Yagoub M C. Development of sensors and microcontrollers for small temperature controller systems. Journal of Automation and Control Engineering, 2015, $3(4): 322-328$

[6] Gaurav S A, Dipesh S V. Design and simulation of temperature control of chamber based $\begin{array}{lllll}\text { on automatic fan } & \text { speed }\end{array}$ http://www.ijaerd.com/papers/special_papers/I1066905.pdf

[7] Bahar A N, Baowaly M K, Chakraborty A. An intelligent approach of regulating electric-fan adapting to temperature and relative humidity. International Journal of Intelligent Systems and Applications, 2012, 4(7):61-69 
[8] Das T K, Das Y. Design of a room temperature and humidity controller using fuzzy logic. American Journal of Engineering Research, 2013, 2(11):86-97

[9] Rizman Z I, Yeap K H, Ismail N, Mohamad N, Husin N H. Design an automatic temperature control system for smart electric fan using PIC. International Journal of Science and Research, 2013, 2(9):1-4

[10] Mounika C H, Roy K S, Ali M. Motor speed control based on temperature using can protocol. International Journal of Engineering Trends and Technology, 2013, 4(4):1249-1252

[11] Pimpalgaonkar A, Jha M, Shukla N, Asthana K. A precision temperature controller using embedded system. International Journal of Scientific and Research Publications, 2013, $3(12): 1-3$

[12] Okpagu P E, Nwosu A W. Development and temperature control of smart egg incubator system for various types of egg. European Journal of Engineering and Technology, 4(2):13-21 [13] Rina A, Zairi I R, Nik N S N D, Syila I I, Rosmawati S, Mohamad H J.Design an automatic temperature control system for smart tudung saji using Arduino microcontroller.ARPN Journal of Engineering and Applied Sciences, 2016, 11(16):9578-9581 [14] Nawi B, Sulaini B, Mohd Z A R, Shamsul A Z, Zairi I R.PID voltage control for DC motor using MATLAB Simulink and Arduino microcontroller. Journal of Applied Environmental and Biological Sciences, 2015, 5(9):166-173

\section{How to cite this article:}

Dzulkefli N N S N, Abdullah R, Jaafar A N, Shafie R, Yassin I M, Rizman Z I, Abidin H Z. Design an automatic temperature control system based on pic controller for smart ventilation fan. $\mathrm{J}$. Fundam. Appl. Sci., 2017, 9(3S), 779-790. 